\title{
Are interacting systems norm asymptotic abelian?
}

\author{
Heide Narnhofer
}

Received: 17 April 2015 / Accepted: 21 July 2015 / Published online: 12 August 2015

(C) Chapman University 2015

\begin{abstract}
We consider infinite Fermi systems for which time evolution is defined as automorphism group. Under appropriate assumptions quasifree evolutions are norm asymptotic abelian on the even subalgebra. If interaction is added in the ground state strong asymptotic abelianess is preserved. In the tracial state, however, it is argued that for appropriate interacting systems even strong asymptotic abelianess fails in first order perturbation theory and only weak asymptotic abelianess seems to be possible. Consequences of different kinds of abelianess are discussed.
\end{abstract}

Keywords Long time behavior · Time clustering

\section{Introduction}

Quantum mechanics is expected to manifest itself mainly on the microscopic scale and for few particles. In thermodynamics, it is a general belief that we are well guided by our inspiration from classical mechanics. Of course details like correlation functions and points of space transition need to be treated on the quantum mechanical level. But the idea of approach to equilibrium respectively the mechanism of conductivity based on scattering processes should be similar in quantum mechanics and in classical mechanics. An essential point in this expectation is the assumption that long-time correlations should disappear both in classical mechanics and in quantum mechanics. Are these correlations only properties of the state or do they appear already on the algebraic level and are expressed that asymptotically the commutators vanish?

We have to search for control on the long-time behavior of thermodynamic systems in order to understand approach to equilibrium. Nevertheless, our knowledge for realistic systems is rather limited. Well analyzed are quasifree evolutions, for which the time evolution can be described on the one particle level and, therefore, we can inherit our understanding of few body quantum physics. If the one particle Hamiltonian has absolutely continuous spectrum then the spreading of the wave functions on the one particle level leads to norm asymptotic abelianess on the even subalgebra of the Fermi system of infinitely many particles or to strong asymptotic abelianess for quasifree Bosons.

This fact encouraged one to assume norm asymptotic abelianess or strong asymptotic abelianess as a general feature of time evolution also in a more general setting [1-3] and study its consequences with respect to thermody-

H. Narnhofer $(\bowtie)$

Fakultät für Physik, Universität Wien, Boltzmanngasse 5, 1090 Vienna, Austria

e-mail: heide.narnhofer@univie.ac.at 
namical behavior. The main advantages of this assumption are the facts, that time invariant states can be decomposed uniquely into extremal time invariant states, that in time invariant factor states the state is clustering with respect to time, and that equilibrium states can be described as states that are passive [4] or dynamically stable [5]. For norm asymptotic abelian systems operators after a long evolution commute, because they escape to infinity or are so much delocalized so that they have no influence on localized operators. However we have various possibilities of convergence of the commutator. A natural generalization possible on the algebraic level is G-abelianess, where we have to take invariant means of the operators. An other generalization lies in the difference of convergence. We can assume norm convergence on the algebraic level, but we can also be satisfied with strong convergence or even with weak convergence, for which some kind of non-commutativity remains, that however disappears in the expectation values. This convergence can depend on the representation of the algebra and, therefore, can depend on the state. In fact for all the desired results, i.e. unique decomposition into extremal invariant states, dynamical stability and passivity it is sufficient to assume weak asymptotic abelianess [6].

In this paper, we collect results on asymptotic abelianess. Weak asymptotic abelianess has the advantage that it can be proven for systems that are Galilei invariant [7] or Poincare invariant [8] under some additional assumptions, that however do not seem to be too demanding. The essential tool was the fact, that Galilei invariance or Poincare invariance allows the comparison of the time evolution with space translations, for which we know, that the automorphism group is norm asymptotically abelian. However, the relation between time evolution and space translations was not powerful enough to get control over the norm convergence of the time evolution but only over weak convergence.

But we also look for support for the assumption that for interacting systems only weak asymptotic abelianess is satisfied and not strong asymptotic abelianess or norm asymptotic abelianess. This is motivated by the fact, that dynamical stability also for quasifree evolution holds only for equilibrium states, whereas we can easily construct other invariant states that look perfectly acceptable from a physical point of view.

We expect that weak asymptotic abelianess without strong asymptotic abelianess might reduce the possibility to construct invariant states, because there exist examples that allow only the tracial state as invariant state and are therefore G-abelian: in $[9,10]$ a system with a discrete automorphism group was constructed which under appropriate assumptions allows only the tracial state as an invariant state and is weakly asymptotically abelian but not strongly asymptotic abelian [12,13]. In [11] we constructed another model that is a bit more general and again allows only the tracial state as invariant state in which the automorphism is again weakly asymptotically abelian. The main feature seemed to be that multicommutators do not vanish and therefore the construction of an invariant state demands correlations between far apart subsystems that according to the monotonicity of entanglement cannot be satisfied except for the tracial state that is not entangled at all. From the view point that interaction should reduce the class of time invariant states we might consider the failure of norm asymptotic abelianess as an advantage.

Considering now time evolution in interacting systems already in the construction of the time evolution as a sequence in time, we observe differences between quasifree evolutions and evolutions with interaction. For quasifree evolution, this sequence converges for all times; whereas for interacting systems, the contributions of multi-commutators increase and allow only a finite convergence radius. If we leave the algebraic level and examine the behavior in the ground state, then the time evolution is determined as for finitely many particles [14,15] and with appropriate assumptions the time evolution is strongly asymptotically abelian. If, however, we consider the system in the tracial state where all automorphisms are unitarily implemented and leave the tracial state invariant so that we can compare the unitaries implementing the automorphisms as in scattering theory, we have to notice on the basis of perturbation theory that the multi-commutators, which do not vanish for interacting systems, destroy the norm convergence and in fact interacting systems on the level of perturbation seem to fail to be strongly asymptotically abelian.

The paper is organized as follows: in Sect. 2, we give the necessary definitions of asymptotic abelianess and GNS representation, especially of the tracial state. In Sect. 3, we present the models we have in mind, i.e. Fermions on the lattice, Spin systems and Galilei invariant Fermi systems. We also present the models of [9-11]. In Sect. 4, we discuss the consequences of asymptotic abelianess with respect to the characterization of equilibrium states as well as with respect to scattering theory for automorphisms. In Sect. 5 we recall the known results, i.e., norm asymptotic 
abelianess for the even part of Fermi systems under quasifree evolutions and strong asymptotic abelianess for interacting Fermi systems in the ground state. In Sect. 6, we move to the tracial state and show that under the assumption, that the anticommutator of the creation and annihilation operators vanishes in norm in the limit $t \rightarrow \infty$ it seems that we run into a contradiction for interacting systems. To be more precise we take the interaction as perturbation and find a contradiction in first order of the coupling constant. In Sect. 7 we offer an example where by adding a perturbation to the discrete shift the asymptotic behavior changes. In Sects. 8 and 9 we examine how the relations between a two-dimensional algebra and its shift vary for strong and weak asymptotic abelian evolution and their indication for invariant states. In the conclusion, we refer once more how weak and strong asymptotic properties are of different relevance depending whether we are in a ground state or in a state with separating GNS vector as in the tracial state.

\section{The various kinds of asymptotic abelianess}

We follow the description in $[2,3]$. The theory is developed in the framework of a $C^{*}$ algebra $\mathcal{A}$ with a time evolution given by an automorphism group $\tau_{t}, t \in R$ and states $\omega$ over the algebra. We are interested in the correlations between operators $A, \tau_{t} \mathrm{~B}$ for $t \rightarrow \pm \infty$. These correlations can be expressed on the algebraic level or with respect to the states. These states can be controlled by the GNS construction:

Theorem 1 The GNS construction: Let $\omega(A)$ be a state over $\mathcal{A}$. Then there exists a cyclic representation on a Hilbertspace $H_{\omega}$ a vector $|\Omega\rangle$ in $H_{\omega}$ and a representation $\pi_{\omega}(A)$ of $\mathcal{A}$ in the bounded operators over $H_{\omega}$ such that

$\omega(A)=\left\langle\Omega\left|\pi_{\omega}(A)\right| \Omega\right\rangle$

with $\pi_{\omega}(A)|\Omega\rangle$ dense in $H_{\omega}$

This representation is unique up to isomorphisms.

$\pi_{\omega}(\mathcal{A})$ is, therefore, a subalgebra of all bounded operators $\mathcal{B}\left(H_{\omega}\right)$ and in general allows a commutant $\pi_{\omega}(\mathcal{A})^{\prime \prime}$ of all operators commuting with $\pi_{\omega}(\mathcal{A})$. If the commutant is trivial, then the representation is irreducible. A typical example for such a representation is a physical system in its groundstate. If the representation is separable, i.e., if

$\pi_{\omega}(A)|\Omega\rangle=0 \rightarrow \pi_{\omega}(A)=0$

then Tomita-Takesaki theory applies and the state defines a modular automorphism group $\hat{\tau}_{t}$ such that for the subset of operators that are analytic in $\mathrm{t}$ (this subset is dense in the weak closure $\pi(\mathcal{A})^{\prime \prime}$ of $\pi(\mathcal{A})$ )

$\pi_{\omega}(A)|\Omega\rangle=J \tau_{i / 2} \pi_{\omega}\left(A^{\dagger}\right)|\Omega\rangle=A^{\prime}|\Omega\rangle$

for a unique operator in the commutant with $\|A\|=\left\|A^{\prime}\right\|$. The so defined operator $J$ is antiunitary and satisfies $J^{2}=1$. If $\omega$ is the tracial state

$\omega(A B)=\omega(B A)$

then the corresponding modular automorphism group is trivial.

The GNS construction is a generalization of the Hilbert Schmidt representation of finite dimensional algebras where the density matrix determining the state corresponds to

$$
\begin{aligned}
|\Omega\rangle & \left.=|\sqrt{\rho}\rangle, \quad \pi_{\omega}(A)|\Omega\rangle=A \otimes 1|| \sqrt{\rho}\right\rangle=|A \sqrt{\rho}\rangle \\
A^{\prime}|\Omega\rangle & =1 \otimes A^{\prime}|\sqrt{\rho}\rangle=\left|\sqrt{\rho} A^{\prime \mathrm{T}}\right\rangle \\
\rho & =\mathrm{e}^{-\hat{H}}, \quad \hat{\tau}_{t} A=\mathrm{e}^{i t \hat{H}} A \mathrm{e}^{-i t \hat{H}}
\end{aligned}
$$

where $A^{\prime \mathrm{T}}$ is the transposed of $A^{\prime}$ and the transposed is given with respect to the eigenvectors of $\rho$. It generalizes this representation also in the sense that for equilibrium states up to the appropriate scaling the modular automorphism coincides with the time evolution. The tracial state corresponds to infinite temperature. We can now express the various kinds of asymptotic abelianess: 
Normasymptotic abelian:

$\mathrm{n}-\lim _{t \rightarrow \pm \infty}\left[A \tau_{t} B\right]=0$

Strong asymptotic abelian:

$\operatorname{st}_{t \rightarrow \pm \infty} \pi_{\omega}\left[A \tau_{t} B\right]=0$

We can demand that this holds in all representations. But we can also reduce the class of representation we are interested in and in fact it will turn out this is necessary.

Weak asymptotic abelian

$\underset{t \rightarrow \pm \infty}{\mathrm{W}-\lim _{\omega}} \pi_{\omega}\left[A \tau_{t} B\right]=0$

Again we can reduce the class of representations we are interested in. Especially we can reduce it to states that are invariant with respect to the automorphism group.

G-abelianess

$\eta \omega\left(\left[A, \tau_{t} B\right]\right)=0$

where $\eta$ is some invariant mean over the amenable group or as stronger demand runs over all invariant means.

It is clear that norm asymptotic abelianess $\rightarrow$ strong asymptotic abelianess $\rightarrow$ weak asymptotic abelianess $\rightarrow \eta$-abelianess.

\section{The models}

The model for which we have the strongest results concerning time evolution is the Fermi algebra on the lattice. The algebra is built by creation and annihilation operators $a_{x}, a_{y}^{\dagger}, x, y \in Z^{v}$ satisfying $a_{x} a_{y}+a_{y} a_{x}=0, a_{x} a_{y}^{\dagger}+a_{y}^{\dagger} a_{x}=$ $\delta_{x y}$. The time evolution is implemented by a Hamiltonian

$H_{\Lambda}=\sum_{x, y \in \Lambda}\left[\phi(x-y) a_{x}^{\dagger} a_{y}+\alpha v(x-y) a_{x}^{\dagger} a_{y}^{\dagger} a_{y} a_{x}\right]$

$\tau_{t}(A)=\lim _{\Lambda \rightarrow Z^{\nu}} \mathrm{e}^{i t H_{\Lambda}} A \mathrm{e}^{-i t H_{\Lambda}}$

and under appropriate assumptions [3] this limit exists. If $v(x-y)=0$ then the time evolution is free and can be written as

$\tau_{t} a(f)=a\left(\mathrm{e}^{i t \phi} f\right)$

Anticommutators between creation and annihilation operators reduce to $\left\langle f\left|\mathrm{e}^{i t \phi}\right| g\right\rangle$ and vanish asymptotically, if $\phi$ has absolutely continuous spectrum. Then the even algebra is norm asymptotically abelian.

Adding an interaction, i.e., a quartic term we can observe a difference between quasifree evolution and time evolution with interaction already in the construction of the time evolution, considering the time evolution as a powerseries in $t$. For quasifree evolution the limit $\Lambda \rightarrow \infty$ exists for all $t$, whereas for interacting systems the series has only a finite radius of convergence, though we can extend the time evolution for all $t$ because this radius is independent of the chosen $A$ [3]. Generalizations for the Hamiltonian are possible, but we want to concentrate on the simplest examples that already show the relevant features. No general results on the asymptotic behavior exist.

The next model that appears is the spin lattice model. It is built by the spin matrices at different points $\sigma_{\alpha, j}, \alpha=$ $x, y, z ; j \in Z^{v}$ with the commutation relations

$\left[\sigma_{\alpha j}, \sigma_{\beta k}\right]=i \delta_{j k} \epsilon_{\alpha \beta \gamma} \sigma_{\gamma j}$

Now the time evolution is implemented by a Hamiltonian

$H_{\Lambda}=\sum_{j, k \in \Lambda} \sum_{\alpha, \beta} v(\alpha, \beta, j-k) \sigma_{\alpha j} \sigma_{\beta k}+\sum_{j \in \Lambda} \sum_{\gamma} g_{\gamma} \sigma_{\gamma j}$ 
Again the time evolution is well defined as limit of power series, and again the permitted Hamiltonians can easily be extended to other polynomials. However our choice covers the XY-model

$H_{\Lambda}=\sum_{j \in \Lambda} v\left[\sigma_{x, j} \sigma_{x, j+1}+\sigma_{y, j} \sigma_{y, j+1}\right]+\sum_{j \in \Lambda} g \sigma_{z, j}$,

for which an explicit solution of the time evolution exists [16]. Without interaction $(v=0)$, the time evolution remains strictly local and therefore periodic, but as shown in [16] the additional interaction can turn the time evolution on the even subalgebra, i. e., the algebra built by $\sigma_{z, j}$ and even polynomials in $\sigma_{x, j}, \sigma_{y, j}$ into one similar to a quasifree time evolution. Therefore, the evolution is norm asymptotically abelian on this even subalgebra. The time evolution can be controlled on the total algebra by a crossed product construction that is explicit enough to show that it fails to be norm asymptotic abelian for the total algebra but is weakly asymptotically abelian because invariant states have to be even.

As a last example, we offer a Galilei invariant time evolution on a continuous Fermi system. Now the algebra is built by creation and annihilation operators $a(f), a^{\dagger}(f), f \in L^{2}\left(R^{v}\right)$ with the anticommutator $\left[a(f), a^{\dagger}(g)\right]_{+}=$ $\langle f \mid g\rangle,[a(f), a(g)]_{+}=0$. Point interaction does not define an automorphism on the algebra since it uses in its definition unbounded operators that are defined in the Fock representation but do not belong to the algebra. Already in the Fock representation, it is necessary for a well defined time evolution that the interaction is stable, i. e. that the kinetic energy cannot increase to infinity on the cost of the potential energy. This is only possible for at most one sign of interaction. Convergence of the time series defining the time automorphism can be controlled only independent of the sign of the coupling constant. Therefore, applying this method it is necessary to cut off the interaction between particles whose velocity differs very much. For stable interactions we expect that such a cutoff should be close to reality. This was done in [14] by considering a Hamiltonian

$\left.H=\frac{1}{2 m} \int \mathrm{d} x \nabla_{x} a^{\dagger}(x) \nabla a(x)+\int \mathrm{d} p \mathrm{~d} p^{\prime} \mathrm{d} q \mathrm{~d} q^{\prime} a_{p q}^{\dagger} a_{p^{\prime} q^{\prime}}^{\dagger} v\left(p-p^{\prime}, q-q^{\prime}\right)\right) a_{p^{\prime} q^{\prime}} a_{p q}$

Here $a_{p q}=a(W(p, q) f)$ are annihilation operators smeared with an $f$ that is translated by the Weyl operators $W(p, q)=\mathrm{e}^{i(q P+p X)}$. As concrete example we took in [14] $f$ as a Gauss function so that with appropriate normalization in three dimensions

$a_{p q}=\pi^{-\frac{3}{4}} \int \mathrm{d}^{3} x \mathrm{e}^{-\frac{(q-x)^{2}}{2}+i p x} a(x)$

and the convergence is under control very similar as in the Fermi system on the lattice. The advantage of the above model is the fact that it is closer to realistic systems and that the time evolution is related to space translation over Galilei invariance so that it can inherit weak asymptotical abelianess [6]. Galilei invariance connects time, space and gauge transformations and boost

$\sigma_{x} a(f(y))=a(f(x+y)) \quad \gamma_{b} a(f(y))=a\left(\mathrm{e}^{i b y} f(y)\right) \quad v_{\alpha} a(f)=\mathrm{e}^{i \alpha} a(f) \quad \tau_{t}$,

such that

$\sigma_{x} \circ v_{\alpha}=v_{\alpha} \circ \sigma_{x}, \quad \gamma_{b} \circ v_{\alpha}=v_{\alpha} \circ \gamma_{b}, \quad \gamma_{b} \circ \sigma_{x}=\sigma_{x} \circ \gamma_{b} \circ v_{-b x}$

$\tau_{t} \circ v_{\alpha}=v_{\alpha} \circ \tau_{t}, \quad \tau_{t} \circ \sigma_{x}=\sigma_{x} \circ \tau_{t} \tau_{t} \circ \gamma_{b}=\gamma_{b} \circ \tau_{t} \circ \sigma_{b t} \circ v_{-b^{2} t / 2}$

A small boost translation that is controlled in norm connects time translations with space translations. If the clustering with respect to space translation is uniform in the set of slightly boosted states clustering with respect to time translation follows, and this suffices for weak asymptotic abelianess. However, Galilei invariance is not sufficient to give control over multi correlations as it is necessary for strong asymptotic abelianess.

Finally as examples with discrete steps we mention the models of $[9,10]$ where the automorphism $\alpha$ is only discrete:

$\mathcal{A}=\left\{e_{j} ; e_{j}^{2}=1, e_{j} e_{k}=(-1)^{g(j-k)} e_{k} e_{j}, \alpha e_{j}=e_{j+1} ; g(j)= \pm 1\right\}$

The algebra consists of spins that sometimes commute and sometimes anticommute. If $g(j)$ is random then with probability 1 the tracial state $\omega\left(e_{j} \ldots e_{k}\right)=0, \omega(1)=1$ is the only state that is invariant under the shift $[12,13]$. It 
clusters and is therefore weakly asymptotically abelian but not strongly asymptotically abelian. In arbitrary states at least the invariant mean over commutators vanishes. In [11] the operators $e_{j}$ were replaced by the unitary Weyl operators on a lattice and it was possible to construct models where the translated Weyl operators became an increasing product of Weyl operators $W_{\vec{k}}^{p}, \vec{k} \in Z_{d}^{2}=\left\{\left(k_{1}, k_{2}\right) ; k_{i}=0,1, \ldots d-1\right\}$ where $p$ refers to the lattice position and they satisfy the discrete Weyl-like commutation relations

$W_{\vec{k}_{1}}^{p} W_{\vec{k}_{2}}^{p}=\mathrm{e}^{\frac{2 \pi i \sigma\left(\vec{k}_{1}, \vec{k}_{2}\right)}{d}} W_{\vec{k}_{2}}^{p} W_{\vec{k}_{1}}^{p} \sigma\left(\vec{k}_{1}, \vec{k}_{2}\right)=k_{11} k_{22}-k_{12} k_{21}$

The automorphism $\alpha$ is given by

$$
\begin{aligned}
\alpha^{p} W_{\vec{k}}^{0} & =W_{\vec{k}}^{(p)}, \quad W_{\vec{k}_{p}}^{(p)}=W_{l(\overrightarrow{0})}^{0} \otimes W_{l(\overrightarrow{2})}^{2} . . \otimes W_{l(\vec{p})}^{p} \\
W_{\vec{k}_{p}}^{(p)} W_{\vec{k}_{q}}^{(q)} & =\mathrm{e}^{2 \pi i u_{\vec{k}_{p} \vec{k}_{q}}^{(q-p)} W_{\vec{k}_{q}}^{(q)} W_{\vec{k}_{p}}^{(p)},} \\
u_{\vec{k}_{p} \vec{k}_{q}}(q-p) & :=\frac{1}{d} \sigma\left(\vec{k}_{p}, A_{q-p} \vec{k}_{q}\right) .
\end{aligned}
$$

The noncommutativity is now fixed by the random matrix $A_{q-p}$ and again with probability 1 the tracial state is the only invariant state. This implies weak asymptotic abelianess in the tracial state and $\eta$-abelianess in all other states.

In fact, these two examples motivate our interest in the kind of abelianess for realistic time evolutions. The example of Galilei invariant systems suggests, that for translation invariant systems it is reasonable to expect weak asymptotic abelianess. The XY-Model supports this expectation. It also supports the suspicion, that strong or norm asymptotic abelianess cannot be satisfied. In addition in the two last examples operators become delocalized, but the remaining local part acts as a severe restriction on possible invariant states and creates correlations between delocalized regions (delocalized with respect to the normal space translation). Therefore systems that are not norm asymptotic abelian can be weakly asymptotic abelian but are more restrictive in the construction of invariant states. Since we expect that for interacting systems the class of invariant states is much smaller than for free systems, it is worthwhile to look for support of these relations between interaction, weak asymptotic abelianess without strong asymptotic abelianess and the construction of invariant state.

\section{Consequences of asymptotic abelianess}

The main motivation to introduce the concept of asymptotic abelianess was the demand to decompose invariant states into extremal invariant states in a unique way. This holds if the invariant operators that define the decomposition belong to the center of the GNS representation so that the decomposition in extremal states is unique as in the classical situation [1-3]. This is satisfied for all kinds of asymptotic abelianess, starting from G-abelianess (invariant means are taken) over weak asymptotic abelianess up to norm asymptotic abelianess.

In addition however, asymptotic abelianess turned out to be a useful tool to describe special features of equilibrium states with respect to perturbations. Passive states are states for which under local perturbation of the dynamics for a finite time the energy increases. This is a kind to express the second law of thermodynamic. For infinite systems they are equilibrium states due to the fact that space translation is asymptotically abelian. States that are dynamically stable [5] in the sense that a local perturbation of the dynamics though lasting for ever cannot create infinite energy are equilibrium states if the unperturbed dynamics is weakly asymptotically abelian [6].

Strong asymptotic abelianess guarantees return to equilibrium: Let $\omega$ be an invariant state. Then $\lim _{t \rightarrow \infty} \tau_{t} A$ converges to an element in the center. If this center is trivial then

$\lim _{t \rightarrow \infty} \omega\left(B \tau_{t} A B^{\dagger}\right)=\lim _{t \rightarrow \infty} \omega\left(\tau_{t} A\right) \omega\left(B B^{\dagger}\right)$

But for states with GNS representation, that is cyclic and separating weak asymptotic abelianess is sufficient for return to equilibrium.

Norm asymptotic abelianess, however, offers new possibilities. In [3] ideas of scattering theory are introduced for time automorphisms. They are taken up in $[17,18]$ for the construction of invariant states. They consider the 
time evolution $\tau_{t}$ to be perturbed locally by an operator $B$ to $\tau_{t, B}$ so that $\tau_{t, B}$ is the time evolution we are interested in, $B$ describes the Hamiltonian of a finite system and in addition its interaction with a heat bath and $\tau_{t}$ describes the time evolution of the infinite heat bath, respectively, of several heat baths. We are interested how the finite system reacts on the heat baths. Then the effect can be controlled by

$\tau_{t, B} \tau_{-t}(A)=A+i \int_{0}^{t} \mathrm{~d} t^{\prime} \tau_{t^{\prime}, B}\left[B, \tau_{-t^{\prime}} A\right]$

Under the assumption that the commutator vanishes sufficiently fast for $t \rightarrow \infty$ the expression converges and defines under additional assumptions [3] a Moeller automorphism $\gamma_{B}^{ \pm}$depending on the time direction. A state $\omega$ initially invariant under $\tau_{t}$ will turn into $\omega \circ \gamma_{B}^{ \pm}$.

Let us assume as a special example that the time evolution is norm asymptotic abelian. Then we can take, e.g., as perturbation the operator $B$ as $\lim _{\Lambda \rightarrow \infty}\left\{H_{\Lambda}-\left(a(f)+a^{\dagger}(f)\right) H_{\Lambda}\left(a(f)+a^{\dagger}(f)\right)\right\}$. In this case (21) is implemented by $\left(a(f)+a^{\dagger}(f)\right) \tau_{t}\left(a(f)+a^{\dagger}(f)\right)$. Norm asymptotic abelianess implies

$\gamma_{B}^{ \pm}(A)=\gamma\left(a(f)+a^{\dagger}(f)\right)(A)\left(a(f)+a^{\dagger}(f)\right)$

where $\gamma$ is the automorphism $\gamma(A)= \pm A$ depending whether $A$ is even or odd so that the Moeller automorphism do not only exist without additional assumptions but are independent of the time direction.

\section{Control on norm and strong asymptotic abelianess}

For free or quasifree evolution of Fermions everything takes place on the one particle level. If $\phi(x-y)$ considered as operator on $l^{2}\left(Z^{v}\right)$ has absolutely continuous spectrum then

$\lim _{t \rightarrow \infty}\left\|\left[a\left(f_{1}\right), \tau_{t} a^{\dagger}\left(f_{2}\right)\right]_{+}\right\|=\lim _{t \rightarrow \infty}\left\langle f_{1} \mid \mathrm{e}^{i t \phi} f_{2}\right\rangle=0$

Commutators between even polynomials of creation and annihilation operators are linear combinations of anticommutators and vanish therefore in norm. Therefore, on the even subalgebra time evolution is norm asymptotically abelian, whereas the odd algebra is not observable and therefore of no physical relevance.

For the lattice systems except of the XY-model (partly norm-partly weak asymptotic abelian) and Ising type models $[19,20]$, for which however $\sigma_{z}$ remains invariant, no results on the long-time behavior are known to me.

For interacting systems, we know that the time evolution maps the $n$-particle space into the $n$-particle space. If we, therefore, stay in Fock space where the representation is of type I and the time evolution is inner and implemented by e $\mathrm{e}^{i H t}$ we can study the effect of $\tau_{t} a(f)$ on the $n$-particle space and evaluate with $|\Omega\rangle$ the vacuum and $\left|k_{1}, \ldots k_{n}\right\rangle$ an $n$-particle state constructed by the creation operators $a^{\dagger}\left(k_{i}\right)$ where $k_{i}$ are $L^{2}$ functions:

$\tau_{t}(a(f)) a^{\dagger}(k) \Pi_{0 \leq j \leq n} a^{\dagger}\left(k_{j}\right)|\Omega\rangle=\mathrm{e}^{i t H} a(f)\left|k, k_{1}, \ldots k_{n} ;-t\right\rangle$

If on the $n+1$ particle space scattering theory applies then the $n+1$ wave function will have support in a region with negligible intersection with $f$, therefore we can continue to

$(-1)^{n+1} \mathrm{e}^{i t H}\left|k, \ldots k_{n} ;-t, f\right\rangle=(-1)^{n+1}\left|k, \ldots k_{n}, f_{t}\right\rangle=(-1) a^{\dagger}(k) \tau_{t} a(f)\left|k_{1}, \ldots k_{n}\right\rangle$

so that in the limit the creation and annihilation operators will anticommute. How fast they will reach this limit depends on the wave function on which they act and therefore we only have strong asymptotic abelianess. The above consideration was suggested in [14] and carried through with more care and more explicit estimates in [15]. There it was shown that the method applies for the Hamiltonian (14) in three dimensions (the spreading of the wave functions enters in the estimates), but that it also works, if we have point interaction and therefore a time evolution that is only well defined for the von Neumann algebra in the groundstate but not for the $C^{*}$ algebra. The estimates depend heavily on the fact that we apply scattering theory and that the scattering process is well localized. Responsible for strong asymptotic abelianess is the fact, that particles become asymptotically free and localized wave functions spread in the course of time. Since with increasing number of particles, the wave functions become less localized at the beginning the time till they are sufficiently spread so that anticommutativity applies, increases 
with the number of particles. This makes it evident that the method is only applicable in the ground state and cannot be generalized to temperature states with infinitely many particles.

\section{The tracial state}

The essential idea to control long-time behavior is often scattering theory or more generally using its strategy, replacing the limit as an integral over the derivative and treating the derivative on a perturbative level. We want to examine whether

$\lim _{t \rightarrow \infty}\left[\tau_{t} a(f), a^{\dagger}(k)\right]_{+}=0$

in the strong or in the norm sense. From this fact, we could inherit asymptotically abelianess for the even algebra. According to the results in $[7,8]$ we assume that the commutator converges at least weakly. Therefore also the individual terms in the anticommutator tend weakly to a $\mathrm{C}$-number. This $\mathrm{C}$-number corresponds with the expectation value in the tracial state and is therefore 0 . Taking into account that $a(f)+a^{\dagger}(f)$ is unitary and

$\underset{t \rightarrow \infty}{\mathrm{w}-\lim _{t \rightarrow \infty}} a(f)+a^{\dagger}(f)=0$

the convergence can only be weakly. If the anticommutator tends to 0 strongly or in norm, this asks, therefore, for a delicate canceling of the terms. As we will see, the possibility of such a cancelation is rather restricted in the tracial state.

We concentrate on the Fermi algebra on the lattice. Generalization to the Galilei invariant Fermi algebra are obvious. However, we assume inspired by the result for Galilei invariant systems that the time evolution is weakly asymptotic abelian also in this case.

We assume we are in the tracial state $(4) \omega(A B)=\omega(B A)$. On the Hilbertspace of the GNS representation of this state there act apart from $a(f), a^{\dagger}(g)$ and its polynomials also $J a(f) J=c^{\dagger}(f)$ and its adjoints where $J$ as defined in (3) is antiunitary with $J|\Omega\rangle=|\Omega\rangle$ and $J A|\Omega\rangle=A^{\dagger}|\Omega\rangle$ for all $A$, that are polynomials in $a(f), a^{\dagger}(g)$. These additional operators commute with $a(f), a^{\dagger}(g)$, i.e. they form the commutant of the algebra, and they inherit multiplication rules form the algebra, i.e. they anticommute $\left[c(f), c^{\dagger}(g)\right]_{+}=\langle g \mid f\rangle$. In addition

$a(f)|\Omega\rangle=c^{\dagger}(\bar{f})|\Omega\rangle$

We have to take into account that in a temperature state or in the tracial state $(\beta=0)$ the time evolution is not implemented by an operator of the algebra but by an operator renormalized with the corresponding contribution of the commutant such that $H=-J H J$ with $J$ the modular conjugation, and that a similar construction is possible for all automorphisms. For all these operators the GNS vector $|\Omega\rangle$ is invariant. This makes it possible to apply ideas of scattering theory not only to the automorphisms but also to the unitaries that implement the automorphisms.

Since the time evolution is implemented by a Hamiltonian satisfying $H=-J H J, \mathrm{e}^{i H t}$ implements the time evolution also on the commutant with $J \tau_{t} A J=\tau_{t} J A J$.

Let us define

$C_{1}=a_{0}+a_{0}^{\dagger} \quad C_{2}=c_{0}+c_{0}^{\dagger} \quad C=C_{1} C_{2}$

where we indicate by the index whether the operator belongs to the algebra or to the commutant. It follows from the commutation relations that $C_{1}^{2}=C_{2}^{2}=C^{2}=1$. They are also selfadjoint and therefore unitary. As remarked in (23) $C_{1}$ and $C_{2}$ only converge weakly to 0 . However under the assumption that the time evolution on the annihilation operators is either strongly asymptotically abelian or strongly asymptotically antiabelian (the sign depends whether $\mathrm{A}$ is an even or an odd operator or if we have asymptotic abelianess for all operators) in the tracial representation it follows that for all $A$ of the algebra

$\underset{t \rightarrow \infty}{\mathrm{W}-\lim _{t}} \tau_{t} C A|\Omega\rangle= \pm A \tau_{t} C|\Omega\rangle= \pm A|\Omega\rangle$ 
Since $A|\Omega\rangle$ is dense in the Hilbertspace, by the regularization of the operator with its counterpart in the commutant we have turned weak convergence of $\tau_{t} C_{12}$ for $\tau_{t} C$ into strong convergence (remember $C$ is unitary)

$\operatorname{st~}_{t \rightarrow \infty} \mathrm{e}^{i H t} C \mathrm{e}^{-i H t}=W$

with $W$ defined by

$W|\Psi\rangle= \pm|\Psi\rangle$

for even resp. odd vectors $|\Psi\rangle=A|\Omega\rangle$, where $A$ are either even or odd polynomials in the creation and annihilation operators.

The argument can be inverted. Assume

$\operatorname{st~}_{t \rightarrow \infty} \mathrm{e}^{i H t} C \mathrm{e}^{-i H t}=W$

Then

st $\lim _{t \rightarrow \infty}\left[\mathrm{e}^{i H t} C \mathrm{e}^{-i H t}, A\right]_{ \pm} B|\Omega\rangle=[W, A]_{ \pm} B|\Omega\rangle$

implies

$$
\begin{aligned}
& \operatorname{st} \lim _{t \rightarrow \infty}\left[\tau_{t}\left(c_{0}+c_{0}^{\dagger}\right)\left(a_{0}+a_{0}^{\dagger}\right), A\right]_{ \pm}|\Omega\rangle=0=\operatorname{st}_{t \rightarrow \infty}\left[\tau_{t}\left(a_{0}+a_{0}^{\dagger}\right), A\right]_{ \pm}|\Omega\rangle \\
& \text { st } \lim _{t \rightarrow \infty}\left[\tau_{t}\left(a_{0}+a_{0}^{\dagger}\right), A\right]_{ \pm}=0
\end{aligned}
$$

where we use the fact that $\left(c_{0}+c_{0}^{\dagger}\right)$ is unitary and in the last argument that $|\Omega\rangle$ is separating for $\mathcal{A}$.

Therefore, we have to look for arguments in support for strong convergence or against it. To do this we construct an extended Fock-space built by creation and annihilation operators belonging to the algebra and the commutant and such that $|\Omega\rangle$ is its vacuum state. This works with defining

$b(f)=W c(f)$

$b(f)$ anticommutes with $a(g)$. With the Bogoliubov transformation

$A(f)=a(f)-b^{\dagger}(\bar{f}) \quad B(f)=b(f)+a^{\dagger}(\bar{f})$

Strong asymptotic anti-abelianess for $a(f)$ implies the same for $b(f)$ and by linear combination for $A(f)$ and $B(f)$. We have to evaluate how the Hamiltonian can be expressed by $A(f), B(f)$. First we concentrate on the quasifree part and for simplicity consider creation and annihilation operators on a lattice.

$\left(A_{x}^{\dagger}-B_{x}\right)\left(A_{y}-B_{y}^{\dagger}\right)-\left(B_{x}^{\dagger}+A_{x}\right)\left(B_{y}+A_{y}^{\dagger}\right)=A_{x}^{\dagger} A_{y}-B_{x} A_{y}+B_{x} B_{y}^{\dagger}-A_{x}^{\dagger} B_{y}^{\dagger}-A_{x} A_{y}^{\dagger}-A_{x} B_{y}-B_{x}^{\dagger} B_{y}-B_{x}^{\dagger} A_{y}^{\dagger}$

Summation over $x, y$ leads to

$H_{q f}=\sum_{x, y} \phi(x-y)\left(A_{x}^{\dagger} A_{y}-B_{x}^{\dagger} B_{y}\right)$

Therefore, the quasifree timeevolution in the tracial state can be considered as a quasifree time evolution in the enlarged Fock space where the system is clearly separated in a part where the energy is positive and a part where it is negative.

Calculating the contributions to the quartic term, we observe that we get a mixture of creation and annihilation operators that is of order 4 but not gauge invariant any more with respect to the gauging of the extended Fock space (see the appendix)

Considering the consequences for abelianess, we observe that the quasifree time evolutions preserve the number operator with respect to $A(f)$, respectively, with respect to $B(f)$. As in Fock space strong convergence of the anticommutator of odd operators is equivalent to the fact that for the annihilation operators in the enlarged algebra $A(f), B(f)$

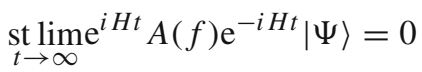


This strong convergence of $\tau_{t} A$ for quasifree evolutions follows, because because weak asymptotic abelianess implies weak convergence of $\mathrm{w}-\lim _{t \rightarrow \infty} \mathrm{e}^{i H t}=0$ in every subspace with given numberoperator $N_{A}, N_{B}$ and orthogonal to $|\Omega\rangle$. Now, we observe that with an appropriate decomposition into time invariant subspaces the annihilation operator acts as a compact operator, so that it turns weak convergence into strong convergence. This decomposition is also possible in the ground state of interacting systems if the scattering operator is sufficiently localized, and therefore in the ground state interacting systems are strongly asymptotically abelian. However this mechanism does not work any more for interacting systems in the extended Fock space of the tracial representation and $A(f)$. Now the Hamiltonian is not gauge invariant any more with respect to $N_{A}, N_{B}$, and no other division into subspaces seems to be available, in which we could control how weak convergence could be turned into strong convergence. Therefore the spreading of $\mathrm{e}^{i H t}|\Psi\rangle$ in an infinite dimensional space (as a consequence of weak convergence) with increasing particle number can be taken as indication, that the time evolution for interacting systems is not strongly asymptotically abelian.

This does, however, give no definite answer. Therefore we look for other arguments and start to study directly the time behavior of $\tau_{t} C$ and look whether its convergence can be easier controlled, since we can understand the interaction as a perturbation of the quasifree evolution. We write

$\tau_{t} C=\int_{0}^{t} \mathrm{~d} t^{\prime} \tau_{t^{\prime}} i[H, C]+C$

and expand the time evolution of (9) in the coupling constant $\alpha$. All information is already contained in

$\left[a^{\dagger}(g), \tau_{t}\left(C_{1} C_{2}\right)\right]_{ \pm}$

and corresponding expressions with $a^{\dagger}, b^{\dagger}$. For the quasifree time evolution we obtain

$\left[a^{\dagger}(g), \tau_{t}\left(C_{1} C_{2}\right)\right]_{+}=0$

and therefore an expansion in $\alpha$ can only lead to 0 if we expand the anticommutator. Such an expansion corresponds to

$\lim _{t \rightarrow \infty} \lim _{\alpha \rightarrow 0} \frac{1}{\alpha}\left[a^{\dagger}(g), \tau_{t}^{(\alpha)}(a(f) b(f))\right]_{+}$

whereas we are really interested in

$\lim _{\alpha \rightarrow 0} \lim _{t \rightarrow \infty} \frac{1}{\alpha}\left[a^{\dagger}(g), \tau_{t}^{(\alpha)}(a(f) b(f))\right]_{+}$

The exchange of the limits is not under control, especially when we take into account that the time evolution as a power series in $\alpha$ is only guaranteed for sufficiently small $\alpha$ and $t$. If however (37) does not converge we take it as a support that also (38) has no chances to converge, especially because for perturbations by quasifree evolutions, we can control that the corresponding expression converges.

In first order in $\alpha$, we want to control if and in which sense

$\int_{0}^{t} \mathrm{~d} t^{\prime}\left[\tau_{t^{\prime}}^{0} V, \tau_{t}^{0}\left(C_{1} C_{2}\right)\right] A_{1}|\Omega\rangle$

tends to 0 . Since the unperturbed timeevolution is norm asymptotic abelian this equals to

$\left(1-\tau_{t}^{0}\left(C_{1} C_{2}\right)\right) \int_{0}^{t} \mathrm{~d} t^{\prime} \tau_{t^{\prime}}^{0} V A_{1}|\Omega\rangle$

Further we know that $V|\Omega\rangle=0$ such that we have to evaluate

$\left(1-\tau_{t}^{0}\left(C_{1} C_{2}\right)\right) \int_{0}^{t} \mathrm{~d} t^{\prime}\left[\tau_{t^{\prime}}^{0} V, A_{1}\right]|\Omega\rangle$

$C_{2}$ is a unitary that cannot change strong convergence, further it commutes with $\left[\tau_{t^{\prime}}^{0} V, A_{1}\right]$ and satisfies $C_{1}|\Omega\rangle=$ $C_{2}|\Omega\rangle$. Combining these facts we end up with

$\left[\tau_{t}^{0}\left(C_{1}\right), \int_{0}^{t} \mathrm{~d} t^{\prime}\left[\tau_{t^{\prime}}^{0} V, A_{1}\right]\right]|\Omega\rangle$ 
which is an equation expressed only by operators belonging to the algebra and therefore could have been obtained directly without applying the correction terms of the commutant. Again we can observe a difference between quasifree evolution and evolution with interaction. We can take $A_{1}$ to be, e.g., a creation operator in the algebra. If $V$ is quasifree, i. e., of order 2 then the double commutator is a C-number for which all topologies coincide. If however $V$ corresponds to an interaction then the double commutator is an operator of order 2 and we have to control whether the vector converges to 0 in strong or in weak topology or not at all. We become more explicit and concentrate on

$$
\begin{aligned}
& i \int_{0}^{t} \mathrm{~d} t^{\prime}\left[\tau_{t}^{0} a^{\dagger}(g),\left[\tau_{t^{\prime}}^{0} \sum_{x, y} v(x-y) a_{x}^{\dagger} a_{y}^{\dagger} a_{y} a_{x}, a(f)\right]\right] \\
& \quad=i \int_{0}^{t} \mathrm{~d} t^{\prime} \int \mathrm{d} u \mathrm{~d} p \mathrm{~d} q \mathrm{~d} r \mathrm{~d} s\left[a^{\dagger}(u), \tilde{v}(r)\left[a_{p}^{\dagger} a_{q}^{\dagger} a_{q-r} a_{p+r}, a(s)\right]\right] g(u) f(s) \mathrm{e}^{\left(i \left(t \phi(u)+t^{\prime}(\phi(p)+\phi(q)-\phi(q-r)-\phi(p+r))\right.\right.}
\end{aligned}
$$

where we have moved into momentum space where the quasifree unperturbed timeevolution is described by $\phi(p)$. The time integration over $t^{\prime}$ leads essentially to $\delta$-functions with a contribution concentrated at $p=q-r$. This contribution gives

$$
\begin{aligned}
& \int \tilde{v}(r) g(u) f(s)\left[a_{u}^{*},\left[a_{p}^{*} a_{p} a_{p+r}^{*} a_{p+r}, a_{s}\right]_{-}\right]_{+} \mathrm{d} p \\
& =\tilde{v}(r) g(u) f(s)\left[a_{u}^{*}, a_{s-r}^{*} a_{s-r} a_{s}+a_{s} a_{s+r}^{*} a_{s+r}\right]_{+}
\end{aligned}
$$

For functions $g$ and $f$ concentrated such that $\left[a_{u}^{*}, a_{s}\right]_{+}=0$ we remain with

$$
\left.\int g(u) f(s)\left(\tilde{v}(s-u) a_{u}^{*} a_{s}+\tilde{v}(u-s) a_{u}^{*} a_{s}\right)\right) \mathrm{e}^{i \phi(u) t} \mathrm{~d} u \mathrm{~d} s
$$

i.e. an operator that weakly tends to 0 but not strongly. The argument depends heavily on $\phi(p)$. The quasifree evolution must delocalize the interaction term. We can consider the counterexample

$\tau_{0}^{n} a_{x}=a_{x+n} \quad V=\alpha \sum_{x} a_{x}^{*} a_{x} a_{x+1}^{*} a_{x+1}$

Then we choose as perturbed automorphism $\tau_{\alpha}=\tau_{0} \circ \gamma_{\alpha}=\tau_{\alpha}=\gamma_{\alpha} \circ \tau_{\alpha}$ such that $\tau_{\alpha}^{n}=\tau_{0}^{n} \circ \gamma_{\alpha}^{n}$ where

$\gamma_{\alpha}^{n} a_{x}=\mathrm{e}^{i \alpha n\left(a_{x-1}^{*} a_{x-1}+a_{x+1}^{*} a_{x+1}\right)} a_{x}$

and acts only locally. Therefore,

$\tau_{\alpha}^{n} a_{x}=\mathrm{e}^{i \alpha n\left(a_{x+n-1}^{*} a_{x+n-1}+a_{x+n+1}^{*} a_{x+n+1}\right)} a_{x+n}$

and the automorphism is norm asymptotically antiabelian, respectively, abelian. Also in the previous formula, we observe that the integration over $t^{\prime}$ just gives $t$ which does not destroy the convergence of the anticommutator.

Returning to (45) also in the ground state this operators appears on the perturbative level. But it can be controlled by the effect of the annihilation operators.

We return to the question whether it is plausible that with $C=\left(a_{0}-a_{0}^{*}\right)\left(b_{0}+b_{0}^{*}\right)$ and taking into account that $[H, W]=0$

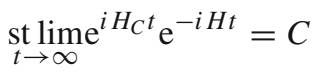

This property only makes sense in the tracial state and we examine it in the extended Fock space. First we calculate

$C=\left(a_{0}-a_{0}^{*}\right)\left(b_{0}+b_{0}^{*}\right)=1+A_{0}^{*} A_{0}+B_{0}^{*} B_{0}-B_{0}^{*} A_{0}-A_{0}^{*} B_{0}$

Writing $H_{C}=H+C\left[H_{0}, C\right]+C[V, C]=H+\tilde{V}$ we obtain the local operator $\tilde{V}$. Scattering theory works in general if $\mathrm{e}^{i H t} \tilde{V} \mathrm{e}^{-i H t}$ tends strongly to 0 . Otherwise it would ask for a delicate cancelation of different terms. $C\left[H_{0}, C\right]$ is an operator in creation and annihilation operators and well ordered, therefore it converges strongly 
to 0 provided the anticommutator of odd operators vanishes asymptotically. It remains to look for the structure of $C[V, C]$ and here we have a contribution of

$\left[A_{0}^{*} B_{0}^{*}, C\right]=-2 A_{0}^{*} B_{0}^{*}$

which does not converge strongly. This leads again to a contradiction on the perturbative level. On the non perturbative level only a delicate cancelation between different contributions could save strong asymptotic abelianess.

Summarizing the ideas of this chapter we have looked for different arguments in the tracial state that might support strong asymptotic abelianess. Extension to the total algebra of bounded operators in the Hilbertspace of the GNS representation allows to describe the system as in Fock space. But in this description for interacting systems gauge invariance as important tool fails. The advantage, that the convergence of commutators can be replaced by the convergence of special unitaries (24) also indicates, that on the perturbative level for interaction gauge invariance is violated. This can be taken as a hint that strong asymptotic abelianess can hardly hold for interacting systems, whereas weak asymptotic abelianess still is a plausible assumption.

If we want to apply these ideas also to spin systems we have to start with a time evolution that is under control. Fortunately the results of [16] offer such a possibility: we can consider the interaction on the lattice system as a perturbation of the $X Y$ model for which we know its asymptotic behavior on the even algebra that is isomorphic to the even Fermi algebra. Of course again the question remains, how far we are allowed to use perturbation theory for the additional interaction and to rely that the asymptotic behavior can be controlled on the level of the expansion parameter.

\section{Instability under perturbation}

So far we have seen that there are essential differences between quasifree evolutions and evolutions with interaction. However this is only a hint that we are not allowed to expect strong asymptotic abelianess. How this strong asymptotic abelianess might be turned over into weak asymptotic abelianess by a perturbation is shown in the following example. We start with an algebra

$\mathcal{A}=\otimes_{i-\infty}^{+\infty} \mathcal{B}_{i} \otimes C_{i}$

where both $\vec{\sigma}_{i} \in \mathcal{B}_{i}, \vec{\tau}_{i} \in \mathcal{C}_{i}$ are $M_{2}$ matrix algebras and $\vec{\sigma}, \vec{\tau}$ are Pauli matrices. On $\mathcal{A}$ acts the shift

$\alpha \vec{\sigma}_{i}=\vec{\sigma}_{i+1}, \quad \alpha \vec{\tau}_{i}=\vec{\tau}_{i-1}$

which is norm asymptotically abelian. In addition we define the automorphism $\gamma$

$\gamma \sigma_{i}^{z} \otimes 1=\sigma_{i}^{z} \otimes \tau_{i+1}^{z}, \quad \gamma 1 \otimes \tau_{i}^{z}=1 \otimes \tau_{i}^{z}$

$\gamma \sigma_{i}^{y} \otimes 1=\sigma_{i}^{y} \otimes \tau_{i+1}^{z}, \quad \gamma 1 \otimes \tau_{i}^{y}=\sigma_{i-1}^{x} \otimes \tau_{i}^{y}$

$\gamma \sigma_{i}^{x} \otimes 1=\sigma_{i}^{x} \quad \gamma 1 \otimes \tau_{i}^{x}=\sigma_{i-1}^{x} \otimes \tau_{i}^{x}$

Their combination leads to

$(\alpha \circ \gamma)^{2 n} \sigma_{i}^{z}=\sigma_{i+2 n}^{z} \otimes_{k=0}^{2 n} \tau_{i+2 n-1+2 k}^{z}$

and similar expressions for the other operators. We observe that the shift wins in one component whereas in the other component a term $\tau_{i}^{z}$ remains and destroys norm asymptotical abelianess. Weak asymptotic abelianess is still satisfied because the action of $\sigma_{i+2 n}^{z}$ maps a GNS vector to an orthogonal vector.

It was essential to take a shift that maps $\mathcal{B}$ and $\mathcal{C}$ into different directions. If the shift moves both parts into the same direction $\alpha \circ \gamma$ remains norm asymptotically abelian. However also for interacting systems particles move in various directions and change this direction in a scattering process. Therefore, the above example reflects some of the actually expected behavior.

Note, however, that the violation of norm asymptotic abelianess in our example is only poor:

$(\alpha \circ \gamma)^{2 n} \sigma_{i}^{z} \sigma_{i+2}^{z}=\sigma_{i+2 n}^{z} \sigma_{i+2 n+2}^{z}$ 
and commutes in the limit with every localized operator. There the algebra contains a large subalgebra on which the perturbed automorphism is still norm asymptotically abelian, very much, as the even subalgebra for the XY-model is norm asymptotically abelian.

\section{Conditions for asymptotic abelianess}

There is another shortage in our previous model:

$\left[(\alpha \circ \gamma)^{n} \sigma_{0}^{z}, \sigma_{0}^{y}\right]=i \sigma_{0}^{x} \otimes \tau_{2 k}^{z}$

is a unitary operator that cannot converge strongly to 0 in any representation. For interacting systems however it was proven that the time evolution is strongly asymptotically abelian in the Fock representation. How can the behavior depend on the representation?

We concentrate on lattice models where at every lattice point the algebra is two dimensional. Further we use the result of [21,22], that under appropriate assumptions on the Hamiltonian time evolution has finite speed so that up to an arbitrary small error in norm

$\alpha_{t} A \in \mathcal{A}_{[-v t-l, v t+l]} \forall A \in \mathcal{A}_{[-l,+l]}$

Let us write

$\mathcal{A}_{[-v t-1, v t+1]}=\mathcal{M} \otimes \mathcal{B}$

Then up to an negligible error for $\vec{\sigma} \in \mathcal{M}$, the starting two-dimensional subsystem,

$\alpha_{t} \vec{\sigma}=\left(\begin{array}{cc}\cos V & \sin U V \\ -U^{*} \sin V & U^{*} \cos U V\end{array}\right) \vec{\sigma}\left(\begin{array}{cc}V^{*} \cos & -V^{*} \sin U \\ V^{*} U^{*} \sin & V^{*} U^{*} \cos U\end{array}\right)=\vec{\sigma}_{t}$

where $U$ and $V$ are unitaries in $\mathcal{B}$ and cos, sin positive operators in $\mathcal{B}$ satisfying $\cos ^{2}+\sin ^{2}=1$, all operators depending on $t$. The action of $V$ is an automorphism inside of $\mathcal{B}$ and therefore irrelevant for the commutativity. $\vec{\sigma}$ commutes with $\vec{\sigma}_{t}$ iff $\cos \sin =0$, i.e. if the spectrum of cos consists only of $\{0,1\}$. Norm asymptotical abelianess follows if this spectrum condition is satisfied for the norm limit of $\cos _{t}$, for strong asymptotic abelianess it suffices to consider the strong limit of $\cos _{t}$. If however we only demand weak asymptotic abelianess, then it is sufficient that

$\mathrm{w}-\lim _{t} \cos _{t} \sin _{t} U_{t}=0, \quad \mathrm{w}-\lim _{t}\left(\cos _{t}^{2}-U_{t}^{*} \sin _{t}^{2} U_{t}\right)=0$

This holds especially if, as in the example of the previous section,

$\mathrm{W}-\lim _{t} U_{t}=0, \quad \lim _{t} \cos _{t}^{2}=\lim \sin _{t}^{2}=1 / 2$

which reflects the shift together with a random spreading of the operator. That this spreading can be felt differently in different representations is reasonable.

\section{Conditions for invariance}

The main motivation for these consideration came from the observation, that for the Price Powers shift $[9,10]$ and similarly for the twisted shift [11] uniqueness of the invariant state demanded violation of strong asymptotic abelianess. Invariance of the state now demands that for given $\epsilon$ and $t$ sufficiently large

$\left|\omega(\vec{\sigma})-\omega\left(\vec{\sigma}_{t}\right)\right|\langle\epsilon$

If $\omega$ is considered as a state over $\mathcal{M} \otimes \mathcal{B}$ we express it by the density matrix

$R=\left(\begin{array}{ll}\rho_{11} & \rho_{12} \\ \rho_{21} & \rho_{22}\end{array}\right) \quad \rho_{i j} \in \mathcal{B}$. 
Time evolution given by (55) transforms it into

$$
\begin{aligned}
& \rho_{11}(t)=\cos \hat{\rho}_{11} \cos +\sin U \hat{\rho}_{21} \cos +\cos \hat{\rho}_{12} U^{*} \sin +\sin U \hat{\rho}_{22} U^{*} \sin \\
& \rho_{12}(t)=-\cos \hat{\rho}_{11} \sin U-\sin U \hat{\rho}_{21} \sin U+\cos \hat{\rho}_{12} U^{*} \cos U+\sin U \hat{\rho}_{22} U^{*} \cos U \\
& \rho_{21}(t)=\rho_{12}(t)^{*} \\
& \rho_{22}(t)=U^{*} \sin \hat{\rho}_{11} \sin U-U^{*} \cos U \hat{\rho}_{21} \sin U-U^{*} \sin \hat{\rho}_{12} U^{*} \sin U+U^{*} \cos U \hat{\rho}_{22} U^{*} \cos U
\end{aligned}
$$

with $\hat{\rho}_{j k}=V \rho_{j k} V^{*}$. Invariance asks for

$$
\begin{aligned}
& \operatorname{Tr}\left(\hat{\rho}_{11} \cos ^{2}+\hat{\rho}_{22} U^{*} \sin ^{2} U+\sin \cos U \hat{\rho}_{21}+\sin \cos \hat{\rho}_{12} U^{*}\right)=\operatorname{Tr} \rho_{11}(t)=\operatorname{Tr} \hat{\rho}_{11} \\
& \operatorname{Tr}\left(-\hat{\rho}_{11} \sin U \cos +\hat{\rho}_{12} U^{*} \cos U \cos -\hat{\rho}_{21} \sin U \sin U+\hat{\rho}_{22} U^{*} \cos U \sin U\right)=\operatorname{Tr} \rho_{12}(t)=\operatorname{Tr} \hat{\rho}_{12}
\end{aligned}
$$

There exist always solutions, since we can take $R$ commuting with the unitary that implements the automorphism. However we are interested in the class of states on $\mathcal{M}$ that can be extended to invariant states. Therefore, we are interested how far these equalities implement restrictions on the possible values of $\operatorname{Tr} \rho_{i j}$. It may be instructive to express them for the situation when $\mathcal{B}$ itself is also a two-dimensional matrix. Assume $\alpha_{t} \mathcal{M}=\mathcal{B}$, i.e., the shifted algebra commutes with the initial one. This corresponds to

$$
\cos =\left(\begin{array}{ll}
1 & 0 \\
0 & 0
\end{array}\right) \quad \sin =\left(\begin{array}{ll}
0 & 0 \\
0 & 1
\end{array}\right) \quad U=\left(\begin{array}{ll}
0 & 1 \\
1 & 0
\end{array}\right)
$$

We remain with

$\operatorname{Tr}\left(\hat{\rho}_{11} \cos ^{2}+\hat{\rho}_{22} U^{*} \sin ^{2} U\right)=\operatorname{Tr} \hat{\rho}_{11}$

which for every set $\operatorname{Tr} \rho_{i j}$ has solutions. Only if the corresponding state on $\mathcal{M}$ is pure the solution is unique. Otherwise $R$ varies from a tensorproduct up to a pure state.

As another extremal example where we do not have commutativity we choose

$$
\cos =\left(\begin{array}{cc}
1 / 2 & 0 \\
0 & 1 / 2
\end{array}\right) \quad \sin =\left(\begin{array}{cc}
1 / 2 & 0 \\
0 & 1 / 2
\end{array}\right) \quad U=1 / \sqrt{2}\left(\begin{array}{cc}
1 & 1 \\
-1 & 1
\end{array}\right)
$$

We want to extend a pure state of $\mathcal{M}$ with $\operatorname{Tr} \rho_{11}=0$. This implies that $\rho_{22}=0=\rho_{12}$.

$$
1 / 2=\operatorname{Tr}\left(\hat{\rho}_{11}+\hat{\rho}_{22}\right) \cos \sin =1
$$

is a contradiction so that this state cannot be extended. In the realistic case neither $\mathcal{M}$ and $\alpha_{t} \mathcal{M}$ can be imbedded into a 4-dimensional matrix nor is it sufficient to study the possibility of an extension only for one step. The increasing number of steps will reduce the permitted class of states. If, however, we are interested in the limit $t \rightarrow \infty$ and its consequences for time invariant states we can understand the conditions on the trace of $\hat{\rho}_{j k}$ as weak limit points of corresponding expectation values. If the time evolution is strongly asymptotic abelian the conditions on $\cos _{t}, \sin _{t}, U_{t}$ satisfy convergence that is stable under taking products and therefore correspond to the conditions for $\mathcal{B}$ a two-dimensional commuting matrix. There are no restrictions on the extension of the state from the asymptotic regime, the restrictions are due to the final steps of the time evolution. If, however, the time evolution is only weakly asymptotically abelian especially we can assume that $\mathrm{w}-\lim U_{t}=0$ and the restriction

$\operatorname{Tr}\left(\hat{\rho}_{11} \cos ^{2}+\hat{\rho}_{22} U^{*} \sin ^{2} U\right)=\operatorname{Tr} \hat{\rho}_{11}$

survives and is not trivially fulfilled.

Take as example the Price-Powers shift: $\cos _{n}^{2}$ oscillates between 1,0 and $1 / 2$. Variation over the algebra $\mathcal{A}$ makes it impossible to exclude in the limiting state the contributions of $1 / 2$, if the shift is sufficiently irregular, and in fact the only invariant state is the tracial state corresponding to $\rho_{12}=0, \rho_{11}=1 / 2$. 


\section{Conclusion}

We have collected results on the asymptotic behavior of time evolution expressed in its kind of abelianess that expresses long-time correlations on the algebraic level. We realized that we have to distinguish in which topology we examine these correlations. Based on the fact that in the tracial state strong convergence of commutators of observables can be turned over into strong convergence of special unitaries over the Hilbert space of the GNS representation we have argued that the interactions on a perturbative level change drastically the arguments that allow to control the time behavior. We expressed the operators as polynomials in creation and annihilation operators in a unique way, insisting on well ordering. Different from quasifree evolutions for interacting systems the degree of, e.g., creation operators in these polynomials in the extended algebra changes in time. On the basis of an expansion in the interaction strong asymptotic abelianess on the even algebra in the tracial state is not inherited in general from the quasifree evolution and therefore also not norm asymptotic abelian on the algebraic level: These polynomials include sufficiently many annihilation operators that in the ground state tend to 0 in the strong topology but do not tend strongly to 0 in the tracial state but only weakly.

It should be noted that the calculations are not sufficient to prove or disprove norm asymptotic abelianess on a rigorous level since we concentrate only on the derivative. However the result is in agreement with the possibility that the time evolution is weakly asymptotic abelian, as it is shown for Galilei invariant time evolution [6] or Lorentz invariant time evolution [8].

Asymptotic abelianess is the tool to describe decomposition into extremal time invariant states and to justify the special role of temperature states by the demand of passivity, respectively, dynamical stability. For all these problems weak asymptotic abelianess is sufficient. It is also used to explain return to equilibrium, namely that

$\lim _{t \rightarrow \infty} \omega\left(A \tau_{t} B A^{\dagger}\right) / \omega\left(A A^{\dagger}\right)=\omega(B)$

Notice that for the ground state strong asymptotic abelianess is necessary for return to equilibrium. In states where the GNS vector is separating as in the tracial state already weak asymptotic abelianess is sufficient for return to equilibrium. Therefore the fact that the topology of convergence is state depending still satisfies optimistic physical demands.

Based on the mathematical examples [9-11] of evolutions that are only $\eta$ abelian and for which the only invariant state is the tracial state we studied the difference of convergence for two-dimensional subalgebras and observed that weak asymptotic abelianess allows an algebraic relation between observables and the shifted ones that reduces the class of possible states more than strong asymptotic abelianess. This is a hint that weak asymptotic abelianess should be an important tool for studying and searching states that are invariant but not equilibrium states.

Acknowledgments It is a pleasure to thank G. L. Sewell and W. F. Wreszinski for useful comments.

\section{Appendix}

We evaluate the typical term of a quartic interaction expressed by creation and annihilation operators in the extended Fock space:

$$
\begin{aligned}
a_{x}^{\dagger} a_{x} a_{y}^{\dagger} a_{y}= & \left(A_{x}^{\dagger}-B_{x}\right)\left(A_{x}-B_{x}^{\dagger}\right)\left(A_{y}^{\dagger}-B_{y}\right)\left(A_{y}-B_{y}^{\dagger}\right) \\
= & A_{x}^{\dagger} A_{x} A_{y}^{\dagger} A_{y}+A_{x}^{\dagger} A_{x} B_{y} B_{y}^{\dagger}+A_{x}^{\dagger} B_{x}^{\dagger} A_{y}^{\dagger} B_{y}^{\dagger}+A_{x}^{\dagger} B_{x}^{\dagger} B_{y} A_{y} \\
& +B_{x} A_{x} A_{y}^{\dagger} B_{y}^{\dagger}+B_{x} B_{x}^{\dagger} A_{y}^{\dagger} A_{y}+B_{x} A_{x} B_{y} A_{y}+B_{x} B_{x}^{\dagger} B_{y} B_{y}^{\dagger} \\
& -A_{x}^{\dagger} A_{x} B_{y} A_{y}-B_{x} B_{x}^{\dagger} B_{y} A_{y}+B_{x} A_{x} A_{y}^{\dagger} A_{y}-B_{x} A_{x} B_{y} B_{y}^{\dagger} \\
& -A_{x}^{\dagger} B_{x}^{\dagger} A_{y}^{\dagger} A_{y}-A_{x}^{\dagger} \mathcal{B}_{x}^{\dagger} B_{y} B_{y}^{\dagger}+A_{x}^{\dagger} A_{x} A_{y}^{\dagger} B_{y}^{\dagger}-B_{x} B_{x}^{\dagger} A_{y}^{\dagger} B_{y}^{\dagger}
\end{aligned}
$$


and similarly

$$
\begin{aligned}
b_{x}^{\dagger} b_{x} b_{y}^{\dagger} b_{y}= & \left(B_{x}^{\dagger}+A_{x}\right)\left(B_{x}+A_{x}^{\dagger}\right)\left(B_{y}^{\dagger}+A_{y}\right)\left(B_{y}+A_{y}^{\dagger}\right) \\
& +A_{x} A_{x}^{\dagger} A_{y} A_{y}^{\dagger}+A_{x} A_{x}^{\dagger} B_{y}^{\dagger} B_{y}+B_{x}^{\dagger} A_{x}^{\dagger} B_{y}^{\dagger} A_{y}^{\dagger}+B_{x}^{\dagger} A_{x}^{\dagger} A_{y} B_{y} \\
& +A_{x} B_{x} B_{y}^{\dagger} A_{y}^{\dagger}+B_{x}^{\dagger} B_{x} A_{y} A_{y}^{\dagger}+A_{x} B_{x} A_{y} B_{y}+B_{x}^{\dagger} B_{x} B_{y}^{\dagger} B_{y} \\
& +A_{x} A_{x}^{\dagger} A_{y} B_{y}+B_{x}^{\dagger} B_{x} A_{y} B_{y}+A_{x} B_{x} A_{y} A_{y}^{\dagger}+A_{x} B_{x} B_{y}^{\dagger} B_{y} \\
& +B_{x}^{\dagger} A_{x}^{\dagger} A_{y} A_{y}^{\dagger}+B_{x}^{\dagger} A_{x}^{\dagger} B_{y}^{\dagger} B_{y}+A_{x} A_{x}^{\dagger} B_{y}^{\dagger} A_{y}^{\dagger}+B_{x} B_{x}^{\dagger} B_{y}^{\dagger} A_{y}^{\dagger}
\end{aligned}
$$

We combine the corresponding terms to see how far the combination of terms with $a_{x}$ and their correspondance with $b_{x}$ in the potential can cancel terms that are not gauge invariant with respect to the gauging in the extended Fock space and see that many are harmless but the last eight are not invariant:

$$
\begin{aligned}
A_{x}^{\dagger} A_{x} A_{y}^{\dagger} A_{y}-A_{x} A_{x}^{\dagger} A_{y} A_{y}^{\dagger}=A_{x}^{\dagger} A_{x}+A_{y}^{\dagger} A_{y}-1 \\
A_{x}^{\dagger} A_{x} B_{y} B_{y}^{\dagger}-A_{x} A_{x}^{\dagger} B_{y}^{\dagger} B_{y}=A_{x}^{\dagger} A_{x}-B_{y}^{\dagger} B_{y} \\
A_{x}^{\dagger} B_{x}^{\dagger} A_{y}^{\dagger} B_{y}^{\dagger}-B_{x}^{\dagger} A_{x}^{\dagger} B_{y}^{\dagger} A_{y}^{\dagger}=0 \\
A_{x}^{\dagger} B_{x}^{\dagger} B_{y} A_{y}-B_{x}^{\dagger} A_{x}^{\dagger} A_{y} B_{y}=0 \\
B_{x} A_{x} A_{y}^{\dagger} B_{y}^{\dagger}-A_{x} B_{x} B_{y}^{\dagger} A_{y}^{\dagger}=0 \\
B_{x} B_{x}^{\dagger} A_{y}^{\dagger} A_{y}-B_{x}^{\dagger} B_{x} A_{y} A_{y}^{\dagger}=A_{y}^{\dagger} A_{y}-B_{x}^{\dagger} B_{x} \\
B_{x} A_{x} B_{y} A_{y}-A_{x} B_{x} A_{y} B_{y}=0 \\
B_{x} B_{x}^{\dagger} B_{y} B_{y}^{\dagger}-B_{x}^{\dagger} B_{x} B_{y}^{\dagger} B_{y}=-B_{x}^{\dagger} B_{x}-B_{y}^{\dagger} B_{y}+1 \\
A_{x}^{\dagger} A_{x} B_{y} A_{y}+A_{x} A_{x}^{\dagger} A_{y} B_{y}=\left(2 A_{x}^{\dagger} A_{x}-1\right) B_{y} A_{y} \\
B_{x} B_{x}^{\dagger} B_{y} A_{y}+B_{x}^{\dagger} B_{x} A_{y} B_{y}=\left(2 B_{x}^{\dagger} B_{x}-1\right) A_{y} B_{y} \\
B_{x} A_{x} A_{y}^{\dagger} A_{y}+A_{x} B_{x} A_{y} A_{y}^{\dagger}=B_{x} A_{x}\left(2 A_{y}^{\dagger} A_{y}-1\right) \\
B_{x} A_{x} B_{y} B_{y}^{\dagger}+A_{x} B_{x} B_{y}^{\dagger} B_{y}=A_{x} B_{x}\left(2 B_{y}^{\dagger} B_{y}-1\right) \\
A_{x}^{\dagger} B_{x}^{\dagger} A_{y}^{\dagger} A_{y}+B_{x}^{\dagger} A_{x}^{\dagger} A_{y} A_{y}^{\dagger}=A_{x}^{\dagger} B_{x}^{\dagger}\left(2 A_{y}^{\dagger} A_{y}-1\right) \\
A_{x}^{\dagger} B_{x}^{\dagger} B_{y} B_{y}^{\dagger}+B_{x}^{\dagger} A_{x}^{\dagger} B_{y}^{\dagger} B_{y}=B_{x}^{\dagger} A_{x}^{\dagger}\left(2 B_{y}^{\dagger} B_{y}-1\right) \\
A_{x}^{\dagger} A_{x} A_{y}^{\dagger} B_{y}^{\dagger}+A_{x} A_{x}^{\dagger} B_{y}^{\dagger} A_{y}^{\dagger}=\left(2 A_{x}^{\dagger} A_{x}-1\right) A_{y}^{\dagger} B_{y}^{\dagger} \\
B_{x} B_{x}^{\dagger} A_{y}^{\dagger} B_{y}^{\dagger}+B_{x}^{\dagger} B_{x} B_{y}^{\dagger} A_{y}^{\dagger}=B_{y}^{\dagger} A_{y}^{\dagger}\left(2 B_{x}^{\dagger} B_{x}-1\right)
\end{aligned}
$$

\section{References}

1. Doplicher, S., Kadison, R.V., Kastler, D., Robinson, D.W.: Asymptotically abelian systems. Commun. Math. Phys. 6, 101-120 (1967)

2. Ruelle, D.: Statistical mechanics. Benjamin, Amsterdam (1969)

3. Bratteli, O., Robinson D.W.: Operator algebras and quantum statistical mechanics I and II, New York, Springer (1979, 1981)

4. Pusz, W., Woronowicz, S.L.: Passive states and KMS states for general quantum systems. Commun. Math. Phys. 58, 273 (1978)

5. Haag, R., Kastler, D., Trych-Pohlmeyer, E.B.: Stability and equilibrium states. Commun. Math. Phys. 38, 173 (1974)

6. Narnhofer, H.: Dynamical stability revisited. J. Phys. A Math. Theor. 41, 335211 (2008)

7. Narnhofer, H., Thirring, W.: Galilei invariant quantum field theories with pair interaction. Int. J. Mod. Phys. A 6(17), 2937-2970 (1991)

8. Jaekel, C.D., Narnhofer, H., Wreszinski, W.F.: On the mixing property for a class of states of relativistic quantum fields. J. Math. Phys. 51, 1 (2010)

9. Powers, R.T.: An index theory for semigroups of *-endomorphisms of B(H) and type $I I_{1}$ factors. Can. J. Math. 40, 86 (1988)

10. Price, G.L.: Shifts on type $I I_{1}$ factors. Can. J. Math. 39, 492 (1987)

11. Baumgartner, B., Benatti, F., Narnhofer, H.: Translation invariant states on twisted algebras on a lattice. J. Phys. A Math. Theor. 43, $115301(2010)$ 
12. Narnhofer, H., Stoermer, E., Thirring, W.: C* dynamical systems for which the tensor product formula fails. Ergod. Theory Dyn. Syst. 15, 961 (1995)

13. Narnhofer, H., Thirring, W.: C*dynamical systems that are asymptotically highly anticommutative. Lett. Math. Phys. 35, 145 (1995)

14. Narnhofer, H., Thirring, W.: Mixing properties of quantum systems. J. Stat. Phys. 57, 811 (1989)

15. Jaekel, C.D.: Asymptotic triviality of the Moeller operators in Galilei invariant quantum field theories. Lett. Math. Phys. 21, 343 (1991)

16. Araki, H., Matsui, T.: Ground states of the $X Y$-model. Commun. Math. Phys. 101, 213-245 (1985)

17. Jaksic, V., Ogata, Y., Pillet, C.A.: The Green-Kubo formula and the Onsager reciprocity relations in quantum statistical mechanics. Commun. Math. Phys. 265, 721 (2006)

18. Derezinski, J., Jaksic, V., Pillet, C.A.: Perturbation theory of W* dynamics, Liouvillians and KMs states. Rev. Math. Phys. 15, 447 (2003)

19. Emch, G.G.: Non Markovian Model for approach to equilibrium. J. Math. Phys. 7, 1198 (1966)

20. Wreszinski, W.F.: J. Approach to equilibrium for a class of random models of in finite range. Stat. Phys. 138, 567 (2010)

21. Lieb, E., Robinson, D.W.: Finite group velocity of quantum spin systems. Commun. Math. Phys. 28, 251-257 (1972)

22. Nachtergaele, B., Raz, H., Schlein, B., Sims, R.: Lieb-Robinson bounds for harmonic and anharmonic lattice systems. Commun. Math. Phys. 286, 1079-1098 (2009) 